\title{
NN-Based Approximate Model Control for the EAF Electrode Regulator System
}

\author{
Hongge Zhao \\ Shandong Water Polytechnic, Rizhao 276826, China \\ Correspondence should be addressed to Hongge Zhao; lipower06@163.com
}

Received 25 May 2013; Revised 30 July 2013; Accepted 14 August 2013

Academic Editor: Zhiguang Feng

Copyright (C) 2013 Hongge Zhao. This is an open access article distributed under the Creative Commons Attribution License, which permits unrestricted use, distribution, and reproduction in any medium, provided the original work is properly cited.

\begin{abstract}
This paper proposes a robust adaptive neural network controller (RANNC) for electrode regulator system. According to the characteristics of electrode regulator system, an affine-like equivalent model is first derived. Then, the nonlinear control law is derived directly based on the affine-like equivalent model identified with neural networks, which avoids complex control development and intensive computation. The control scheme is simple enough that it can be implemented on an automotive microcontroller system, and the performance meets the system requirements. The stability of the system is established by the Lyapunov method. Several simulations illustrate the effectiveness of the controller.
\end{abstract}

\section{Introduction}

Electric arc furnaces (EAF) are widely used in the steel industry for melting scrap. The most important part of EAF is the electrode regulator system. Its performance affects not only the power utilisation efficiency of the furnace but also the electrode and refractory wear costs. A fast controller response is required to optimise the power utilisation efficiency, but where overshoot of the required electrode position is caused, the results are increased refractory and electrode wear and also, possibly, the injection of unwanted carbon into the steel in the case of a molten bath. Thus, the controller should be designed to meet the requirements of fast response without overshoot. However, the demands are difficult to accomplish since the electrode regulator system is burdened with strong nonlinearity and strong coupling among three phases. Moreover, the control strategy should be simple enough to be implemented on an automotive microcontroller system, while it has to be robust to plant parameter variations.

Several control strategies have been considered and/or applied to furnaces in the past few decades. As early as 1977, Billings and Nicholson proposed a temperature-weighting adaptive controller [1], which uses ambient arc temperature as an additional control parameter to weigh the error feedback. However, the conditions of continuous temperature measurement are not easy to achieve. Zhizhong and Jian pointed that the arc gain can be estimated by the energy applied into the furnace [2], to avoid the problem of continuous temperature measurement, and proposed an adaptive feedback controller for the electrode regulator system, but they did not give an expression for calculating the arc gain. Several of the existing control designs use a linear model of the process and derive a state feedback decoupling controller [3]; however, the controller is only effective around the operating points and has some limitations in the practical applications. Intelligent methods such as fuzzy control and neural network control were also applied in the electrode regulator system [4-9]. Staib et al. proposed to utilize the neural network to control the electrode, which can learn online during the smelt process $[4,5]$. In [7-9], an adaptive inverse control algorithm based on RBF NN is presented, which identifies real-time decoupling by RBF NN online, but the control law computation is too much and needs a high-speed acquisition and processing system. Variable structure control with the sliding mode is an effective method for the control of the nonlinear plant with a parameter uncertainty, and it has also been applied in the electrode regulator system in [6]; however, the stability analysis of the closed-loop system was not given in [6]. In [10], a direct adaptive controller is designed. However, the computation is 
too much and the control scheme is difficult to be applied in practice.

The principal aim of this paper is to present a practiceoriented robust adaptive neural network controller for electrode regulator system. The control strategy is achieved based on approximate model method and the Taylor expansion technology, which avoids complex control development and intensive computation. So, it is easily implemented in practice. Moreover, the decoupling among the three phases is also realized and the controller has a good performance with respect to parameters varying and falling scrap, which can be illustrated by simulation results.

The proposed controller is acceptable in engineering practice, as it can meet the following requirements. (1) The proposed control scheme is simple enough that it can be implemented on an automotive microcontroller system for practical application. (2) The performance of the closed-loop system satisfies the requirements, that is, a fast transient response without overshoot. (3) Robustness of the control system with respect to variations of process parameters is required, which can be caused by production deviations and variations of external conditions. (4) Stability of the closedloop system is rigorously established.

This paper is organized as follows. In Section 2, the nonlinear discrete-time dynamics of electrode regulator system is derived. In Section 3, the input-output approximate model is directly derived via the Taylor expansion and nonlinear control law is implemented using NRBFNN modeling. In Section 4, the robustness of the stability is rigorously established by the Lyapunov method. Finally, several simulations and experiments are presented to illustrate the effectiveness of the proposed nonlinear controller.

\section{Model Construction}

As shown in Figure 1, the electrode regulator system of EAF consists of controller, hydraulic system, and EAF main circuit. In this paper, the hydraulic system and the EAF main circuit are considered to be a generalized plant.

2.1. Hydraulic System. Since we focus on the electrode regulator system, the hydraulic system of EAF is approximated as a third-order system as in $[10,11]$. The transfer function can be written as

$$
G_{s}(s)=\frac{b_{0} s+b_{1}}{a_{0} s^{3}+a_{1} s^{2}+a_{2} s} .
$$

2.2. EAF Main Circuit. A typical power supply system of EAF consists of high-voltage power distribution system, EAF transformer, short net, and electric arc. The equivalent circuit of main circuit is presented in Figure 2, where $\dot{U}_{j}, \quad(j=$ $A, B, C)$ is the primary voltage, $\dot{U}_{j},(j=a, b, c)$ is the secondary voltage, $\dot{I}_{j}$, $(j=A, B, C)$ is the primary current, $\dot{I}_{j}, \quad(j=a, b, c)$ is the secondary current, $R_{d}$ is the short net resistance, $X_{d}$ is the short net reactance, and $Z_{\text {jarc }}$, ( $j=$ $a, b, c)$ is the impedance of the arc. In the furnace there is also electrical conduction between the electrodes. However, according to [12], these currents are nearly $1 \%$ of the phase currents and we focus on the electrode regulator system. So, these mutual inductances will be neglected for simplicity, and we assume that the parameters of the three phases are the same to each other.

In order to obtain a simpler arc model, Köhle proposed to represent the arc as an equivalent linear circuit element constituted by a resistive and a reactive part [13]. In his model, Köhle defines the arc reactance $X_{L}$ as a function of its resistance as follows: $X_{L}=a R_{L}+b R_{L}^{2}$, where the first term represents the influences of low frequency fluctuations and the second represents the influences of harmonics. The values of $a$ and $b$ vary during the melting process.

The primary coils and the secondary coils of the EAF transformer are star connected and delta connected, respectively (as shown in Figure 2). According to the Kirchoff's law, we can obtain the electrode-to-neutral currents as follows:

$$
\begin{aligned}
& i_{a}=\left\|\frac{\dot{U}_{A}\left(Z_{k} / 3 n-n Z_{b}\right)-\dot{U}_{B}\left(Z_{k} / 3 n-n Z_{c}\right)}{Z_{K a b c}}\right\|, \\
& i_{b}=\left\|\frac{\dot{U}_{B}\left(Z_{k} / 3 n-n Z_{c}\right)-\dot{U}_{C}\left(Z_{k} / 3 n-n Z_{a}\right)}{Z_{K a b c}}\right\|, \\
& i_{c}=\left\|\frac{\dot{U}_{C}\left(Z_{k} / 3 n-n Z_{a}\right)-\dot{U}_{A}\left(Z_{k} / 3 n-n Z_{b}\right)}{Z_{K a b c}}\right\|,
\end{aligned}
$$

where $Z_{K a b c}=\left(Z_{k} / 3 n-n Z_{a}\right)\left(Z_{k} / 3 n-n Z_{b}\right)+\left(Z_{k} / 3 n-\right.$ $\left.n Z_{a}\right)\left(Z_{k} / 3 n-n Z_{c}\right)+\left(Z_{k} / 3 n-n Z_{b}\right)\left(Z_{k} / 3 n-n Z_{c}\right), Z_{j}=$ $R_{d}+j X_{d}+l_{j} R_{\text {per }}+j\left(a l_{j} R_{\text {per }}+b\left(l_{j} * R_{\text {per }}\right)^{2}\right),(j=a, b, c)$. $R_{d}$ is the short net resistance, $X_{d}$ is the short net reactance, and $n$ is the transformer ratio.

By using the $n$-order approximation method [14], one has $T i_{j}^{(1)}=i_{j}(k+1)-i_{j}(k), T^{2} i_{j}^{(2)}=i_{j}(k+1)-2 i_{j}(k)+i_{j}(k-1)$, $T^{3} i_{j}^{(3)}=i_{j}(k+1)-3 i_{j}(k)+3 i_{j}(k-1)-i_{j}(k-2), \quad(j=a, b, c)$ with $T$ being the sampling period. This way, according to (1) and (2), the generalized control plant can be described in the discrete system as

$$
i(k+1)=f[\omega(k), u(k)],
$$

where $i(k+1)=\left[i_{a}(k+1), i_{b}(k+1), i_{c}(k+1)\right]^{T}$, $\omega(k) \quad=\quad\left[i_{a}(k), i_{a}(k-1), i_{a}(k-\right.$ $\left.2), i_{b}(k), i_{b}(k-1), i_{b}(k-2), i_{c}(k), i_{c}(k-1), i_{c}(k-2)\right]^{T}$, $u(k)=\left[u_{a}(k), u_{b}(k), u_{c}(k)\right]^{T}$, and $f(\cdot)=\left[f_{a}(\cdot), f_{b}(\cdot), f_{c}(\cdot)\right]^{T}$, where $f(\cdot)$ is a vector-valued nonlinear function and $f_{j}(\cdot)$, $(j=a, b, c)$ is regarded as nonlinear mapping.

\section{Control Strategy Design}

In this section, a novel nonlinear controller is proposed based on the approximate method, which avoids complex control development and intensive computation.

3.1. Analysis of the Controlled Object. As $\left\|Z_{K a b c}\right\|>0$ always holds for $Z_{K a b c}$ in (2), it is easy to validate that $f_{j}, \quad(j=a, b, c)$ 


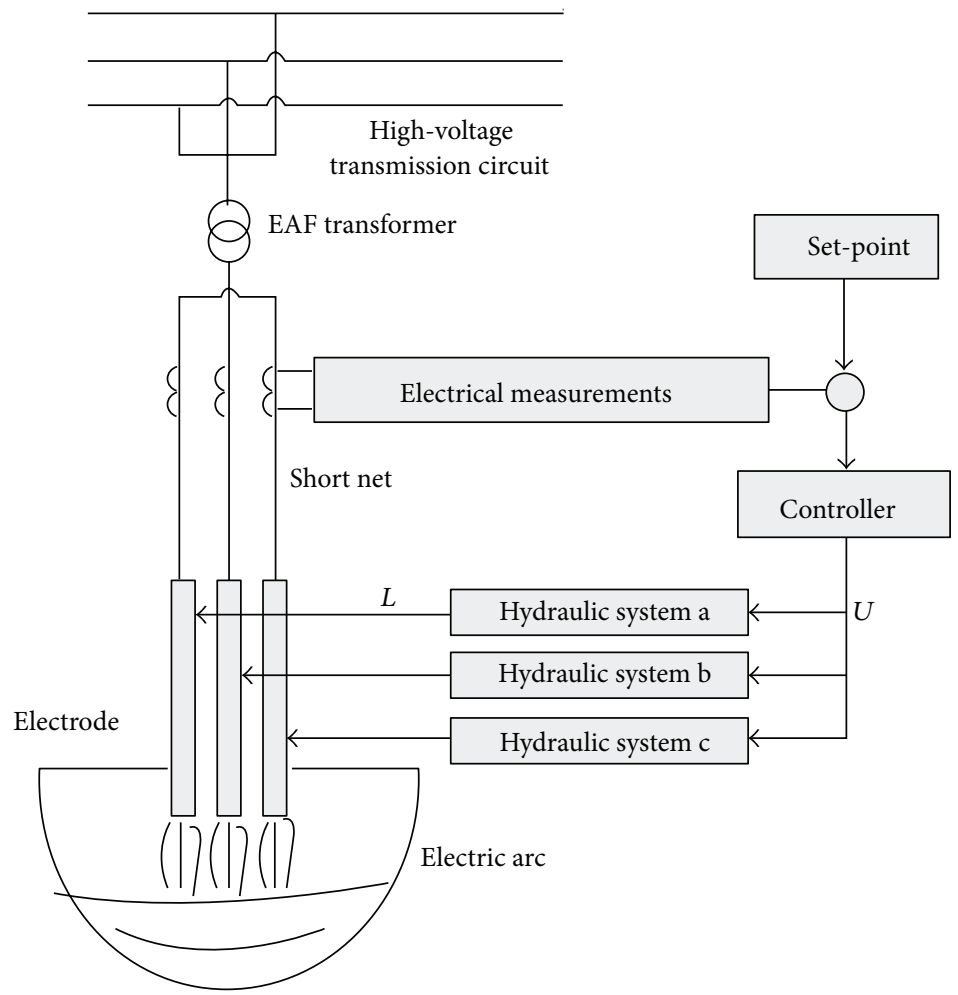

FIGURE 1: Schematic diagram of EAF electrode regulator system.

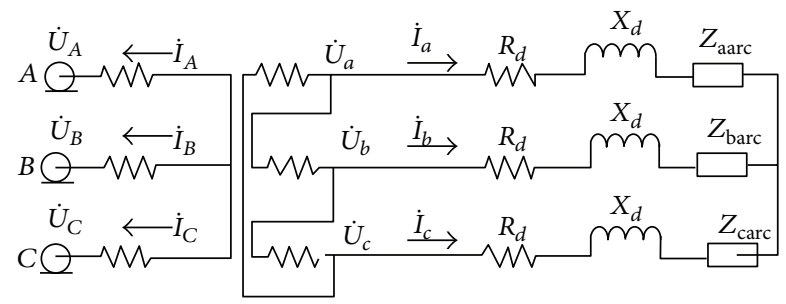

FIgURE 2: Schematic diagram of EAF main circuit.

is derivable. For the nonlinear autoregressive moving average (NARMA) model (3), a Taylor expansion of the plant gives

$$
\begin{aligned}
i_{j}(k+1)= & f_{j}\left[\omega(k-1), u_{j}(k-1)\right] \\
& +\sum \frac{1}{r !} \frac{\partial^{r} f_{j}\left[\omega(k-1), u_{j}(k-1)\right]}{\partial u_{j}^{r}(k-1)}\left[\Delta u_{j}(k)\right]^{r} \\
& +\sum \frac{1}{r !} \frac{\partial^{r} f_{j}\left[\omega(k-1), u_{j}(k-1)\right]}{\partial \omega^{r}(k-1)}[\Delta \omega(k)]^{r},
\end{aligned}
$$

The output $i_{j}(k+1)$ is highly sensitive to control input $u_{j}(k)$ in the operating region; that is, [15],

$$
\begin{array}{r}
\left|\frac{\partial f_{j}\left[\omega(k-1), u_{j}(k-1)\right]}{\partial u_{j}(k-1)}\right| \\
\gg \mid\left(\sum \frac{1}{r !} \frac{\partial^{r} f_{j}\left[\omega(k-1), u_{j}(k-1)\right]}{\partial \omega^{r}(k-1)}\right. \\
\left.\quad \times[\Delta \omega(k)]^{r}\right) \times\left(\Delta u_{j}(k)\right)^{-1} \mid,
\end{array}
$$

where $\Delta$ is the increment operator.

From (5), we can drop the third term on the right-hand side of (4) to represent model (3) by

$$
\begin{aligned}
i_{j}(k+1)= & i_{j}(k)+f_{j}^{1}(k) \Delta u_{j}(k) \\
& +R_{j}\left[\omega(k-1), u_{j}(k-1), \Delta u_{j}(k)\right],
\end{aligned}
$$

where $f_{j}^{1}(k)=\partial f_{j}\left[\omega(k), u_{j}(k)\right] /\left.\partial u_{j}(k)\right|_{u_{j}(k)=u_{j}(k-1), \omega(k)=\omega(k-1)}$, $(j=a, b, c)$.

where $\Delta u_{j}(k)=u_{j}(k)-u_{j}(k-1), \Delta \omega(k)=\omega(k)-\omega(k-1)$, and $(j=a, b, c)$.
Theorem 1 (see [16]). The remainder term $R_{j}\left[\omega(k-1), u_{j}(k-\right.$ 1), $\left.\Delta u_{j}(k)\right]$ in (6) approaches zero at a faster rate than $\Delta u_{j}(k)$ 
approaches zero, and there exists a variable $\tau_{j}(k) \in(0,+\infty]$ such that

$$
\left|\frac{R_{j}\left[\omega(k-1), u_{j}(k-1), \Delta u_{j}(k)\right]}{\Delta u_{j}(k-1)}\right| \ll\left|f_{j}^{1}(k)\right|,
$$

whenever $\left|\Delta u_{j}(k)\right| \in\left[0, \tau_{j}(k)\right],(j=a, b, c)$.

According to the Taylor expansion theory, as $\left|\Delta u_{j}(k)\right| \epsilon$ $\left[0, \tau_{j}(k)\right]$, then the remainder $R_{j}(k)$ in (6) is bounded as follows: $\left|R_{j}(k)\right| \leq 1 / 2 R_{j}^{0} \tau_{j}^{2}(k)$, with $R_{j}^{0}$ being a finite positive number. From (4)-(7), the input-output approximate model of the plant can be derived by neglecting the term $R_{j}(k)$, and thus, (3) can be simplified into

$$
i_{j}(k+1)=i_{j}(k)+f_{j}^{1}(k) \Delta u_{j}(k) \quad(j=a, b, c) .
$$

From (8), the control law can be determined directly since the increment $\Delta u_{j}(k)$ of the control signal appears linearly. Before the computation of the control law, an assumption is given.

Assumption 2. Controller output is bounded; that is, $\left|\Delta u_{j}(k)\right| \leq \delta_{j}(k)$ with $\delta_{j}(k)$ being a finite positive number, which considers constraints on physical variable.

Remark 3. According to Assumption 2, $\Delta u_{j}(k)$ should not be too large in order to limit the approximation error of (8). In electrode regulator system, this is reasonable because the output of the hydraulic system (actuator) cannot change too fast within a small time interval.

3.2. Derivation of Control Law. From (8), it requires precise information about $f_{j}^{1}(k)$ to compute $u_{j}(k)$. However, $f_{j}^{1}(k)$ exists but is unknown. In order to get the nonlinear function $f_{j}^{1}(k)$, a normalized radial basis function neural network (NRBFNN) is used to identify the input-output representation model (3) at first. And then, the nonlinear function $f_{j}^{1}(k)$ can be estimated from the NN model and is referred to as $\widehat{f}_{j}^{1}(k)$.

Evolved from radial basis function neural network (RBFNN), the NRBFNN has the same structure as shown in Figure 3. For the NARMA model (3), the output of the NRBFNN is

$$
\begin{aligned}
\hat{i}_{j}(k+1) & =\overline{n n}\left(\omega(k), u_{j}(k)\right) \\
& =\Theta^{T} \Phi(x)=\sum_{i=1}^{L} \theta_{i} \phi_{i}(x),
\end{aligned}
$$

where $x=\left[\omega^{T}(k), u_{j}(k)\right]$ is the input vector, $\Theta=\left[\theta_{1} \cdots \theta_{L}\right]^{T}$ is the weight vector, and $\Phi(x)=\left[\phi_{1}(x) \cdots \phi_{L}(x)\right]^{T}$ with $\phi_{i}(x)$ being a normalized activation function to node $i$ and being expressed as follows

$$
\phi_{i}(x)=\frac{\exp \left(-\left\|x-C_{i}\right\|^{2} / 2 s^{2}\right)}{\sum_{j=1}^{L} \exp \left(-\left\|x-C_{i}\right\|^{2} / 2 s^{2}\right)},
$$

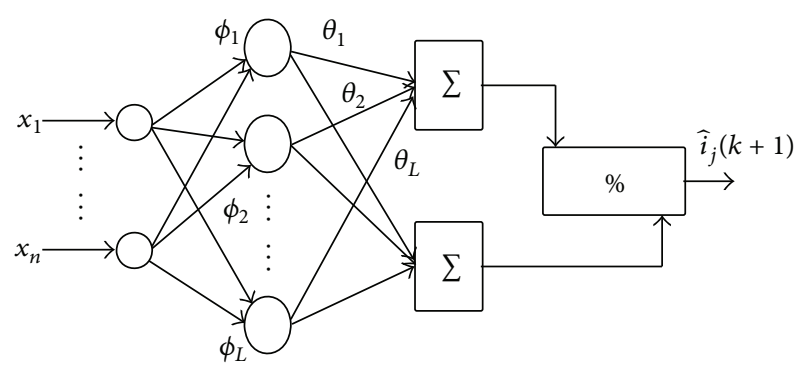

Figure 3: Structure of an NRBFNN.

where $C_{i}=\left[c_{i 1} \cdots c_{i n}\right]$ denotes the centroid vector, $L$ denotes the NN node number, and $s$ is the spread. The NRBFNN can improve function approximation with a minimal number of weights [17]. In (10), the kernel function is very similar to RBF neural network, except that the kernel output is divided by the sum of all the kernel outputs. Therefore, the outputs of all the kernels add up to one; that is, $\sum_{i=1}^{L} \phi_{i}(x)=1$. As the output of every hidden node is less than 1 , we can get that the hidden output vector has the properties $0<\|\Phi(x)\| \leq$ 1. These properties will be used in Section 4 for the stability analysis of the closed-loop system.

To formulate a well-posed adaptive control problem, we make the following assumptions. Similar assumptions are used in $[18,19]$.

Assumption 4 (see [15]). An optimal weight vector $\Theta_{j}^{o}$ for a trained NRBFNN exists, and the corresponding optimal estimation error $e_{j}^{o}(k)=\widehat{i}_{j}^{o}(k)-i_{j}(k)$ has a known finite upper bound $\left[e_{j}^{o}\right]_{\max }$, accordingly

$$
\left|\hat{i}_{j}^{o}(k)-i_{j}(k)\right|=\left|e_{j}^{o}(k)\right|<\left[e_{j}^{o}\right]_{\max } .
$$

Remark 5. The NRBFNN has been theoretically proven to be capable of universal approximation in a satisfactory sense [20]. Assumption 4 shows that a perfect function estimation can be achieved if enough radial basis functions are used. However, more hidden nodes means more computation. There should be a trade-off between the computation burden and approximation errors. In the electrode regulator system, 20 hidden nodes can meet the requirement of the estimation errors.

The parameter update rule must be robust with respect to modeling errors because these errors can prevent the convergence of the weights of the neural network and thereby destabilize the closed-loop system. One of the approaches to ensure convergence of neural network weights is through a "deadzone," which suspends parameter adaption whenever estimation error becomes small. The corresponding weights updated law as follows:

$$
\Theta_{j}(k+1)=\Theta_{j}(k)-\rho_{j}(k) \eta_{j}(k)\left[\frac{e_{j}(k+1) \Phi_{j}(k)}{1+\left\|\Phi_{j}(k)\right\|^{2}}\right],
$$


where $\rho_{j}(k)$ is the adaptive rate, and $0<\rho_{j}(k) \leq\left[\rho_{j}\right]_{\max }<2$, and

$$
\eta_{j}(k)= \begin{cases}1, & \text { if }\left|e_{j}(k+1)\right|>\frac{2\left[e_{j}^{o}\right]_{\max }}{2-\left[\rho_{j}\right]_{\max }}, \\ 0, & \text { otherwise. }\end{cases}
$$

We have a gradient descent parameter update rule with a deadzone, where $e_{j}(k+1)$ is the estimation error at the time $k+1$ and $e_{j}(k+1)=\widehat{i}_{j}(k+1)-i_{j}(k+1)$. Their other parameters such as centroids and spreads can be found in $[18,19]$ and thus are not discussed here. is,

Based on the NRBFNN model (9), we can get $\widehat{f}_{j}^{1}(k)$; that

$$
\widehat{f}_{j}^{1}(k)=\left.\frac{\partial \overline{n n}\left[\omega(k), u_{j}(k)\right]}{\partial u_{j}(k)}\right|_{u_{j}(k)=u_{j}(k-1), \omega(k)=\omega(k-1)} .
$$

And according to Assumptions 2 and 4, the control law can be determined straigthforwardly from (8) as follows:

$$
u_{j}(k)=u_{j}(k-1)+\Delta u_{j}(k), \quad(j=a, b, c),
$$

where

$$
\begin{gathered}
\Delta u_{j}(k)=\frac{r_{j}(k+1)-i_{j}(k)}{\left[\widehat{f}_{j}^{1}(k)\right]^{2}+\alpha} \widehat{f}_{j}^{1}(k) \text { if }\left|\Delta u_{j}(k)\right| \leq \delta_{j}, \\
\Delta u_{j}(k)=\delta_{j} \operatorname{sign}\left|\Delta u_{j}(k)\right| \text { if }\left|\Delta u_{j}(k)\right|>\delta_{j},
\end{gathered}
$$

where $\alpha$ and $\delta_{j}$ are given finite positive constants and $r_{j}(k)$ is the reference current.

Define the tracking error at the time $k$ as

$$
\left[e_{c}\right]_{j}(k)=r_{j}(k)-i_{j}(k), \quad(j=a, b, c) .
$$

\section{Stability Analysis of RANNC}

Stability and performance of the closed-loop system with NN adaptive control (15) and weight updating law (12) are given in Theorem 6.

Theorem 6. For given $\left|r_{j}(k)-r_{j}(k-1)\right| \leq \Delta r$, using the NN control law (15) with NN weight updating law (12), then the solution of the error system (17) is uniformly ultimately bounded (UUB) [21] for all $k$ with ultimate bound $\lim _{k \rightarrow \infty}\left|\left[e_{c}\right]_{j}(k)\right| \leq\left(k_{2} /\left(1-k_{1}\right)\right)$, where $k_{1}=\mid(1-$ $\left.s_{j}(k)\left(\left[\widehat{f}_{j}^{1}(k)\right]^{2} /\left(\left[\widehat{f}_{j}^{1}(k)\right]^{2}+\alpha\right)\right)\right) \mid$ and $k_{2}=k_{1} \cdot r_{0}+2\left[e_{j}^{o}\right]_{\max } /(2-$ $\left.\left[\rho_{j}\right]_{\max }\right)$, in which $0<s_{j}(k) \leq 1, \alpha, r_{0},\left[e_{j}^{o}\right]_{\max }$ and $\left[\rho_{j}\right]_{\max }$ are the same as those defined above, $(j=a, b, c)$.

Proof. See Appendix.

\section{Simulations and Experiment}

5.1. Simulations. This section shows the MATLAB simulations of the proposed approximate model control strategy on the electrode regulator system. The parameter values are selected as follows.
(1) Hydraulic system: $b_{0}=5, b_{1}=95, a_{0}=1, a_{1}=9$, and $a_{2}=110$.

(2) EAF transformer: $\dot{U}_{A}=35000 \mathrm{~V}, \dot{U}_{B}=\dot{U}_{A} e^{-j(2 / 3) \pi}$, $\dot{U}_{C}=\dot{U}_{A} e^{j(2 / 3) \pi}, Z_{K}=(0.0069+j 0.076) \Omega$, and $n=$ 80.

(3) Electric arc: $a=0.12, b=0.02$, and $R_{\text {per }}=$ $0.000058 \Omega / \mathrm{mm}$.

(4) Short net: $\left(Z_{d}=0.0003+j 8.3292 e-006\right) \Omega$.

(5) Controller parameters: $\alpha=0.01,\left[e_{j}^{0}\right]_{\max }=50 \mathrm{~A}, \delta_{j}=$ $3.5 \mathrm{~V}, \rho_{j}=1$, and $s=0.6$.

Here, three controllers' (including the PID controller (PIDC), inverse neural network controller (INNC), Robust adaptive neural network controller (RANNC)) capability of following set-point, restraining parameters varying and falling scrap is studied by simulation.

There are strong coupling among three phases in the electrode regulator system. To validate the decoupling performance of the proposed controller, several set-point trackings are performed with different operating points and reference changes. Figure 4 shows the simulation results of the current $A$ and current $B$. We can know from figures that the RANNC is better in respect of tracking the set value, and the current $B$ is almost not influenced by the changes of current $A$. Approximate decoupling is realized. When the PIDC and INNC are used, the system has slower response and larger overshoot, and its performance is worse than RANNC.

In the smelting process, production deviation and variations of external condition can cause the variation of process parameters, and falling scrap is also common. In order to make the proposed control strategy more acceptable in practice, we simulate these situations and the parameters change at $25 \mathrm{~s}$ and $50 \mathrm{~s}$, respectively. The simulation results are illustrated in Figure 5. We can know from figures that the RANNC preserves important performance measures, like fast response, the little overshoot, and accuracy within the measurement resolution.

Three phase electrodes discharge to molten steel, forming star connection, so there are strong coupling among three phases. In PIDC system, there is three controllers for three phases individually and the coupling effects are not considered. The performance of INNC is better than that of PIDC as the coupling effects are regarded as disturbances in INNC system. However, the complete decoupling is still not achieved. In RANNC system, the control law is directly derived from the approximate model, which fully reflects the coupling effects among the three phases. Approximate complete decoupling can be realized. From Figures 4 and 5, we can get that the performance of the RANNC is better than that of PIDC and INNC.

5.2. Experiment. The experiment apparatus is shown in Figure 6, and our robust adaptive neural network controller is implemented on SIEMENS CPU414-2. The parts of the experiment system include arc model Machine, programmer, HMI, electrode PLC, master PLC, and other models Machine, and they communicate via industry ethernet. Long-distance 


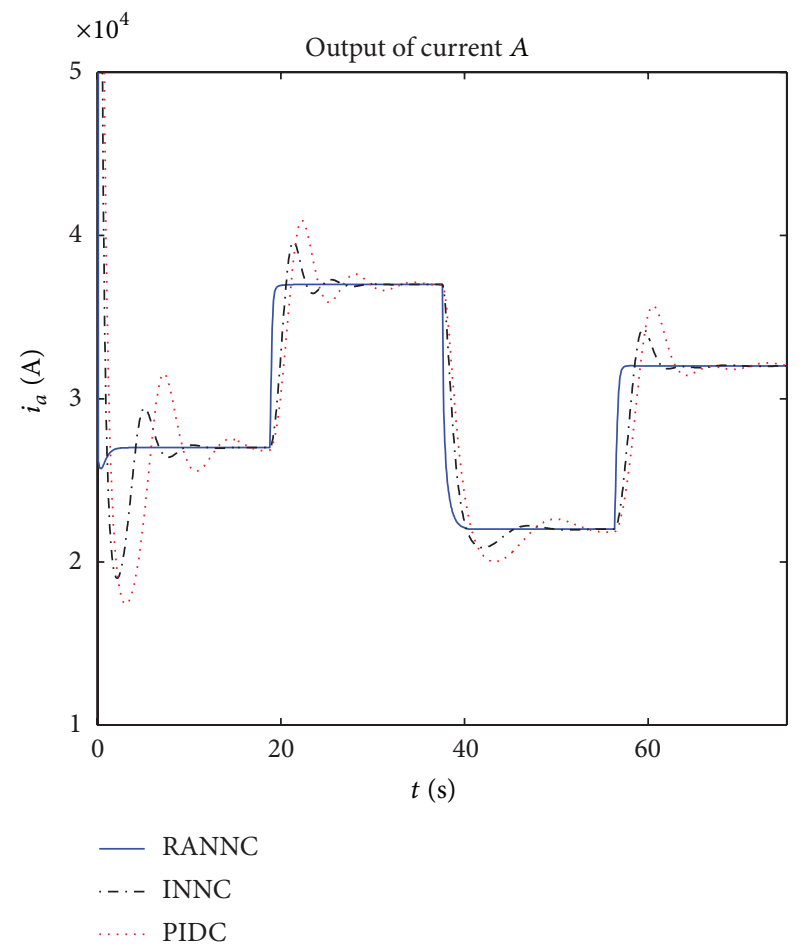

(a)

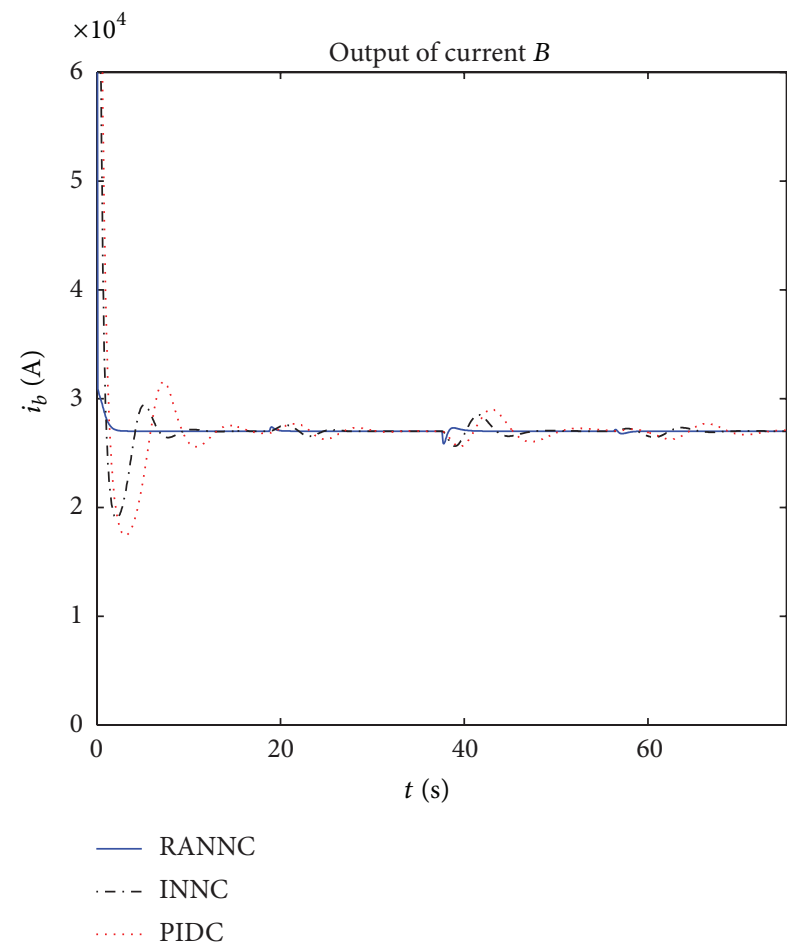

(b)

FIGURE 4: Comparison of decoupling control.

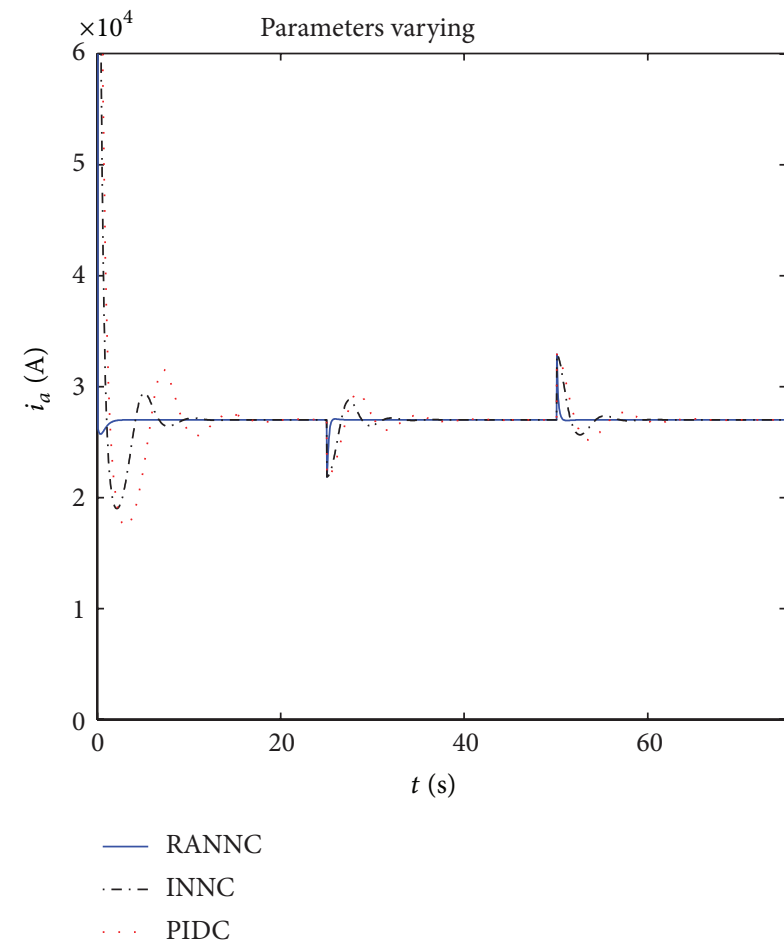

(a)

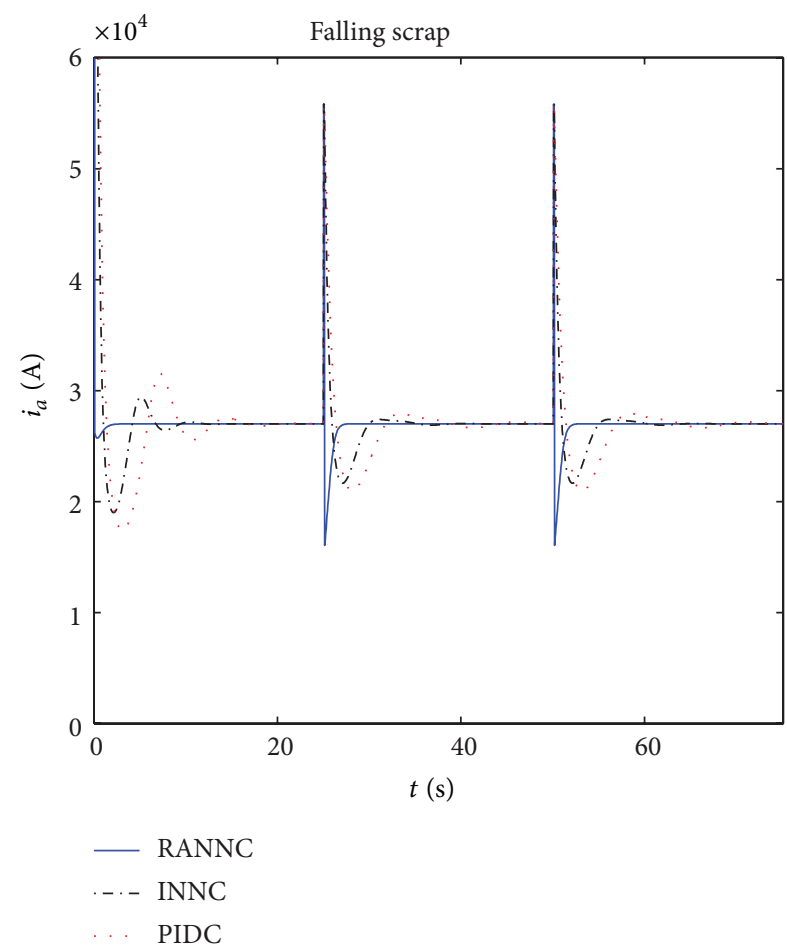

(b)

FIGURE 5: Comparison of robustness and falling scrap. 


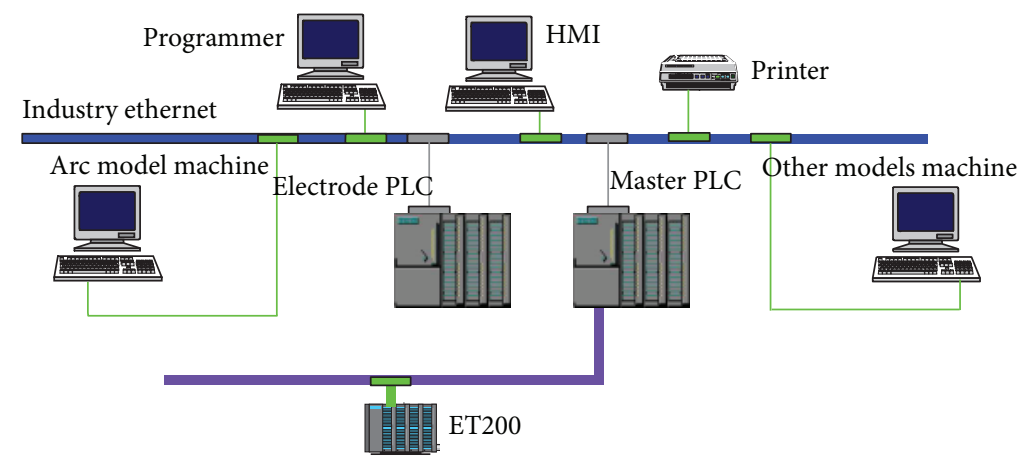

FIGURE 6: Schematic diagram of EAF electrode system.

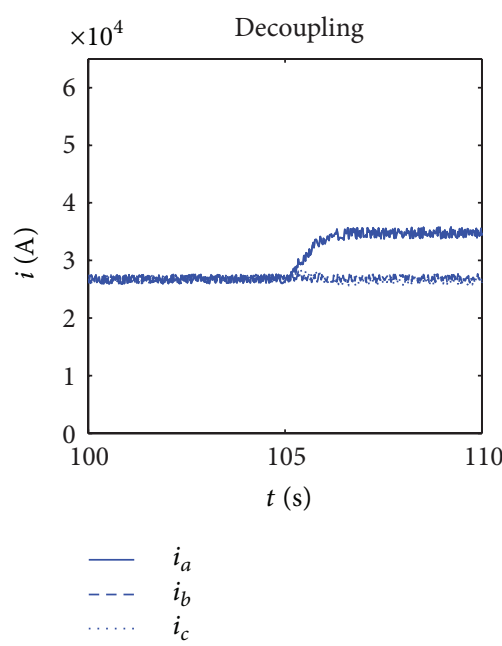

(a)

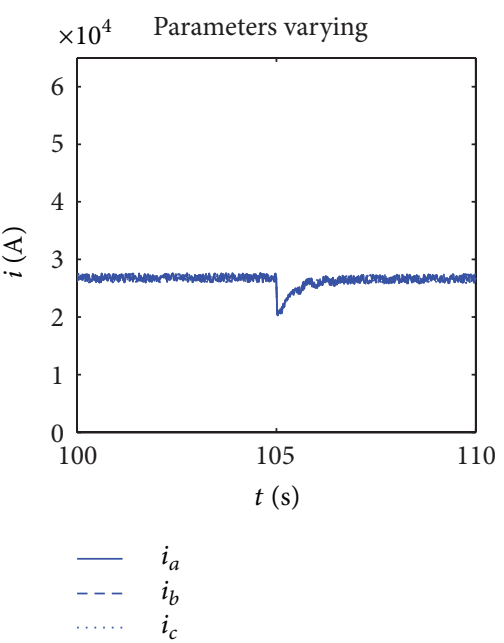

(b)

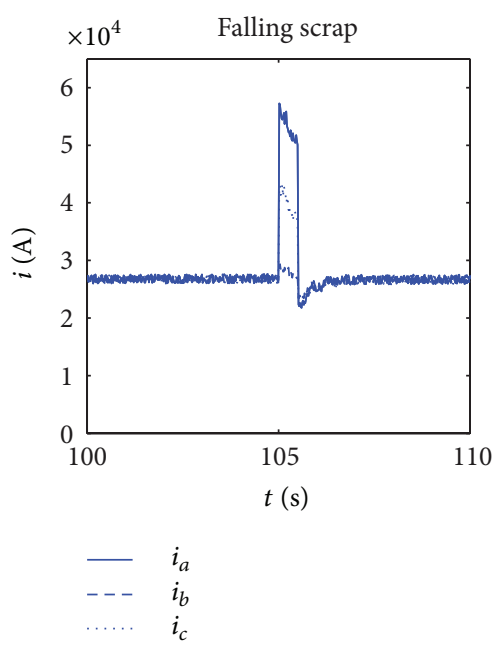

(c)

Figure 7: Output of the currents.

I/O interface ET200M is connected with Master PLC with Profibus DP. The power module is PS407, and the analog input module and analog output module are AI431 and AO432; digital input module and digital output module are DI321 and DO322, respectively. Similar to the simulations, we verify the effectiveness of the controller via decoupling performance, robustness, and antidisturbance ability. The results are shown in Figure 7.

The corresponding parameters are changed at $105 \mathrm{~s}$. Figure 7 shows experimental results of the three phase currents when only RANNC is used. As is shown in Figure 7, the RANNC has a good performance, that is, faster response and smaller overshoot, which means more power utilisation efficiency and less refractory and electrode wear. From the experiments, we can come to the following conclusions.

(i) As the coupling effects by other two phases are considered in the approximate model, the coupling among three phases is decreased greatly. Real-time decoupling and control scheme are realized for the electrode regulator system. (ii) In our proposed controller, small nodes of NRBFNN are chosen, which renders our control scheme possible application in real-time control of electric arc furnace.

\section{Conclusion}

Many nonlinear discrete systems can be described by NARMA model. In this paper, a generalized model for electrode regulator system on NARMA form is established. With a novel $I / O$ approximation proposed for the NARMA model, a robust adaptive controller is derived directly from the approximation being and it can be implemented straightforwardly by using neural network. The controller design method can be also applied on other systems such as electronic throttle valve and distributed curing process etc., in which the approximation model can be derived by the similar procedure. The design of the proposed nonlinear controller is simple and practical. Simulation results illustrate the good performance of this controller. 


\section{Appendix}

We will prove, one by one in the order of the NN weights, the estimation error and the tracking error are bounded.

(1) Boundedness of the weights: Choose a Lyapunov candidate $V_{j}\left(k, \widetilde{\Theta}_{j}(k)\right)=\widetilde{\Theta}_{j}^{T}(k) \widetilde{\Theta}_{j}(k)$, where $\widetilde{\Theta}_{j}(k)=\Theta_{j}(k)-$ $\Theta_{j}^{o}, \Theta_{j}^{o}$ is the optimal weight vector as in Assumption 4; then

$$
\begin{aligned}
\Delta V_{j}\left(k+1, \widetilde{\Theta}_{j}(k+1)\right) & \\
= & V_{j}\left(k+1, \widetilde{\Theta}_{j}(k+1)\right)-V\left(k, \widetilde{\Theta}_{j}(k)\right) \\
& =\widetilde{\Theta}_{j}^{T}(k+1) \widetilde{\Theta}_{j}(k+1)-\widetilde{\Theta}_{j}^{T}(k) \widetilde{\Theta}_{j}(k) .
\end{aligned}
$$

According to [15]

$$
\begin{aligned}
\Delta V_{j}(k & \left.+1, \widetilde{\Theta}_{j}(k+1)\right) \\
= & 2 \widetilde{\Theta}_{j}^{T}(k) \cdot\left[\Delta \widetilde{\Theta}_{j}(k+1)\right] \\
& +\left[\Delta \widetilde{\Theta}_{j}(k+1)\right]^{T} \cdot \Delta \widetilde{\Theta}_{j}(k+1),
\end{aligned}
$$

where $\Delta \widetilde{\Theta}_{j}(k+1)=\widetilde{\Theta}_{j}(k+1)-\widetilde{\Theta}_{j}(k)$, and from (12), we get

$$
\Delta \widetilde{\Theta}_{j}(k+1)=-\rho_{j}(k) \eta_{j}(k)\left[\frac{e_{j}(k+1) \Phi_{j}(k)}{1+\left\|\Phi_{j}(k)\right\|^{2}}\right] .
$$

Note that the estimation error is

$$
\begin{aligned}
e_{j}(k+1) & =\widehat{i}_{j}(k+1)-i_{j}(k+1) \\
& =\widetilde{\Theta}_{j}^{T}(k) \cdot \Phi_{j}(k)+e_{j}^{o}(k+1) .
\end{aligned}
$$

We consider two cases associated with (A.3) separately.

Case $1\left(\left|e_{j}(k+1)\right| \leq 2\left[e_{j}^{o}\right]_{\text {max }} /\left(2-\left[\rho_{j}\right]_{\max }\right)\right)$. Equation (A.3) implies $\widetilde{\Theta}_{j}(k+1)=\widetilde{\Theta}_{j}(k)$, therefore, $\Delta V_{j}\left(k+1, \widetilde{\Theta}_{j}(k+1)\right)=0$.

Case $2\left(\left|e_{j}(k+1)\right|>2\left[e_{j}^{o}\right]_{\max } /\left(2-\left[\rho_{j}\right]_{\max }\right)\right)$. Equation (A.3) implies

$$
\begin{aligned}
\Delta \widetilde{\Theta}_{j}(k+1) & =\widetilde{\Theta}_{j}(k+1)-\widetilde{\Theta}_{j}(k) \\
& =-\rho_{j}(k)\left[\frac{e_{j}(k+1) \Phi_{j}(k)}{1+\left\|\Phi_{j}(k)\right\|^{2}}\right] .
\end{aligned}
$$

From (A.4)

$$
\widetilde{\Theta}_{j}^{T}(k) \cdot \Phi_{j}(k)=e_{j}(k+1)-e_{j}^{o}(k+1) .
$$

Therefore, substituting for $\widetilde{\Theta}_{j}^{T}(k)$ and $\Delta \widetilde{\Theta}_{j}(k+1)$ in (A.2) gives

$$
\begin{aligned}
\Delta V_{j}\left(k+1, \widetilde{\Theta}_{j}(k+1)\right) & 2\left[e_{j}(k+1)-e_{j}^{o}(k+1)\right] \cdot\left[-\rho_{j}(k) \frac{e_{j}(k+1)}{1+\left\|\Phi_{j}(k)\right\|^{2}}\right] \\
+ & {\left[-\rho_{j}(k) \frac{e_{j}(k+1) \Phi_{j}(k)}{1+\left\|\Phi_{j}(k)\right\|^{2}}\right] } \\
& \cdot\left[-\rho_{j}(k) \frac{e_{j}(k+1) \Phi_{j}(k)}{1+\left\|\Phi_{j}(k)\right\|^{2}}\right] .
\end{aligned}
$$

This implies

$$
\begin{aligned}
\Delta V_{j}\left(k+1, \widetilde{\Theta}_{j}(k+1)\right) & \\
=- & \rho_{j}(k)\left[\frac{e_{j}^{2}(k+1)}{1+\left\|\Phi_{j}(k)\right\|^{2}}\right] \\
& \cdot\left[2\left(1-\frac{e_{j}^{o}(k+1)}{e_{j}(k+1)}\right)-\rho_{j}(k) \frac{\left\|\Phi_{j}(k)\right\|^{2}}{1+\left\|\Phi_{j}(k)\right\|^{2}}\right] .
\end{aligned}
$$

Using the fact that $\left|e_{j}(k+1)\right|>2\left[e_{j}^{o}\right]_{\max } /\left(2-\left[\rho_{j}\right]_{\max }\right)$ and $0<\rho_{j}(k) \leq\left[\rho_{j}\right]_{\max }<2$, simple algebraic manipulations of (A.8) can be used to show that $\Delta V_{j}(k) \leq 0$.

Clearly, the system must operate under Cases 1 or 2 or alternate between both cases. Since $\Delta V_{j}(k) \leq 0$, for Cases 1 and 2 , then $\Delta V_{j}(k) \leq 0$ for $k=0,1,2, \ldots$. This implies

$$
V_{j}\left(k, \widetilde{\Theta}_{j}(k)\right)=\left\|\widetilde{\Theta}_{j}(k)\right\|^{2} \leq\left\|\widetilde{\Theta}_{j}(0)\right\|^{2}, \quad \text { for } k=0,1,2, \ldots
$$

This proves that $\widetilde{\Theta}_{j}(k)$ is uniformly bounded. Since $\left\|\widetilde{\Theta}_{j}(k)\right\|$ is uniformly bounded and $\widetilde{\Theta}_{j}(k)=\Theta_{j}(k)-\Theta_{j}^{o}$, where $\Theta_{j}^{o}$ is a constant, then $\left\|\Theta_{j}(k)\right\|$ is uniformly bounded.

(2) Boundedness of the estimation error: since $V_{j}\left(k, \widetilde{\Theta}_{j}(k)\right)$ converges

$$
\lim _{k \rightarrow \infty} \Delta V_{j}\left(k, \widetilde{\Theta}_{j}(k)\right)=0
$$

Clearly, this can only happen if there exists some $k_{0}$, such that

$$
\left|e_{j}(k+1)\right| \leq \frac{2\left[e_{j}^{o}\right]_{\max }}{2-\left[\rho_{j}\right]_{\max }}, \quad \text { for } k=k_{0}, k_{0}+1, k_{0}+2, \ldots
$$

This implies

$$
\widetilde{\Theta}_{j}(k+1)=\widetilde{\Theta}_{j}(k), \quad \text { for } k=k_{0}, k_{0}+1, k_{0}+2, \ldots
$$


which, in turn, implies

$$
\Delta V_{j}\left(k, \widetilde{\Theta}_{j}(k)\right)=0, \quad \text { for } k=k_{0}, k_{0}+1, k_{0}+2, \ldots
$$

The fact that $k_{0}$ exists is proven by contradiction as shown below. If $k_{0}$ described above does not exist, we consider subsequence $t_{k}(i), i=1,2, \ldots$ of instants $k=0,1,2, \ldots$, when weights of the neural network are updated; that is,

$$
t_{k}=\left\{k:\left|e_{j}(k+1)\right|>\frac{2\left[e_{j}^{0}\right]_{\max }}{2-\left[\rho_{j}(k)\right]_{\max }}\right\} .
$$

Under this case,

$$
\begin{aligned}
\Delta V_{j}\left(t_{k}+1, \widetilde{\Theta}_{j}\left(t_{k}+1\right)\right) & \\
= & -\rho_{j}(k)\left[\frac{e_{j}^{2}\left(t_{k}+1\right)}{1+\left\|\Phi_{j}\left(t_{k}\right)\right\|^{2}}\right] \\
& \cdot\left[2\left(1-\frac{e_{j}^{o}\left(t_{k}+1\right)}{e_{j}\left(t_{k}+1\right)}\right)-\rho_{j}(k) \frac{\left\|\Phi_{j}\left(t_{k}\right)\right\|^{2}}{1+\left\|\Phi_{j}\left(t_{k}\right)\right\|}\right] .
\end{aligned}
$$

From (A.8),

$$
\begin{aligned}
V_{j}\left(r, \widetilde{\Theta}_{j}(r)\right)= & V_{j}\left(0, \widetilde{\Theta}_{j}(0)\right) \\
& +\sum_{k=1}^{r}-\rho_{j}(k)\left[\frac{e_{j}^{2}(k+1)}{1+\left\|\Phi_{j}(k)\right\|^{2}}\right] \\
\cdot & {\left[2\left(1-\frac{e_{j}^{o}(k+1)}{e_{j}(k+1)}\right)\right.} \\
\leq & V_{j}(0, \widetilde{\Theta}(0)) \\
& -\lambda \sum_{k=1}^{r}\left[\frac{e_{j}^{2}(k+1)}{1+\left\|\rho_{j}(k)\right\|^{2}}\right] \text { for } \lambda>0 .
\end{aligned}
$$

Hence

$$
\sum_{k=1}^{r} \frac{e_{j}^{2}(k+1)}{1+\left\|\Phi_{j}(k)\right\|^{2}} \leq \frac{1}{\lambda}\left[V\left(0, \widetilde{\Theta}_{j}(0)\right)-V\left(r, \widetilde{\Theta}_{j}(r)\right)\right] .
$$

When $r \rightarrow \infty$, we get

$$
\sum_{k=1}^{\infty} \frac{e_{j}^{2}(k+1)}{1+\left\|\Phi_{j}(k)\right\|^{2}}<\infty
$$

In other words, sequence $e_{j}^{2}(k+1) /\left(1+\left\|\Phi_{j}(k)\right\|^{2}\right)$ converges. Normalized error $e_{j}(k+1) / \sqrt{1+\left\|\Phi_{j}(k)\right\|^{2}}$ is squared summable, and it follows that

$$
\lim _{k \rightarrow \infty} \frac{e_{j}^{2}(k+1)}{1+\left\|\Phi_{j}(k)\right\|^{2}}=0 .
$$

Then, from $0<\left\|\Phi_{j}(k)\right\|^{2} \leq 1$, this implies

$$
\frac{e_{j}^{2}(k+1)}{1+1} \leq \frac{e_{j}^{2}(k+1)}{1+\left\|\Phi_{j}(k)\right\|^{2}}
$$

or

$$
e_{j}^{2}(k+1) \leq 2 \frac{e_{j}^{2}(k+1)}{1+\left\|\Phi_{j}(k)\right\|^{2}}
$$

In the limit as $k \rightarrow \infty$, the right-hand side of (A.21) converges zero which results in

$$
\lim _{k \rightarrow \infty} e_{j}(k)=0
$$

This conclusion clearly contradicts the assumption that $\left|e_{j}\left(t_{k}+1\right)\right|>2\left[e_{j}^{o}\right]_{\max } /\left(2-\left[\rho_{j}\right]_{\max }\right)$ for all $t_{k}$. Therefore, the only possibility is that an integer $k_{0}$ exists such that

$$
\left|\hat{i}_{j}(k+1)-i_{j}(k+1)\right|=\left|e_{j}(k+1)\right| \leq \frac{2\left[e_{j}^{o}\right]_{\max }}{2-\left[\rho_{j}\right]_{\max }}
$$

for $k=k_{0}, k_{0}+1, k_{0}+2, \ldots$ which implies

$$
\lim _{k \rightarrow \infty}\left|e_{j}(k)\right|=\lim _{k \rightarrow \infty}\left|\hat{i}_{j}(k)-i_{j}(k)\right| \leq \frac{2\left[e_{j}^{o}\right]_{\max }}{2-\left[\rho_{j}\right]_{\max }} .
$$

(3) Boundness of the tracking error: define a variable $s_{j}(k)$, where $0<s_{j}(k) \leq 1$ for all $k$. The control law (15) is equivalently expressed as

$$
\Delta u_{j}(k)=s_{j}(k) \frac{r_{j}(k+1)-i_{j}(k)}{\left[\widehat{f}_{j}^{1}(k)\right]^{2}+\alpha} \widehat{f}_{j}^{1}(k),
$$

where $s_{j}(k)=1$ if $\left|\Delta u_{j}(k)\right|<\delta_{j}$, and $0<s_{j}(k)<1$ if $\left|\Delta u_{j}(k)\right|>\delta$. Using (A.25), one has

$$
\begin{aligned}
{\left[e_{c}\right]_{j}(k+1) } & =r_{j}(k+1)-i_{j}(k+1) \\
& =r_{j}(k+1)-i_{j}(k)-f_{j}^{1}(k) \Delta u_{j}(k)
\end{aligned}
$$




$$
\begin{aligned}
= & r_{j}(k+1)-i_{j}(k) \\
& -\widehat{f}_{j}^{1}(k) \Delta u_{j}(k)+e_{j}(k+1) \\
= & r_{j}(k+1)-i_{j}(k) \\
& -s_{j}(k)\left[\widehat{f}_{j}^{1}(k)\right]^{2} \frac{r_{j}(k+1)-i_{j}(k)}{\left[\widehat{f}_{j}^{1}(k)\right]^{2}+\alpha} \\
& +e_{j}(k+1) \\
= & \left(1-s_{j}(k) \frac{\left[\widehat{f}_{j}^{1}(k)\right]^{2}}{\left[\hat{f}_{j}^{1}(k)\right]^{2}+\alpha}\right) \\
& \times\left(r_{j}(k+1)-i_{j}(k)\right)+e_{j}(k+1) \\
= & \left(1-s_{j}(k) \frac{\left[\widehat{f}_{j}^{1}(k)\right]^{2}}{\left[\hat{f}_{j}^{1}(k)\right]^{2}+\alpha}\right) \\
& \times\left(\Delta r_{j}(k+1)+\left[e_{c}\right]_{j}(k)\right)+e_{j}(k+1) .
\end{aligned}
$$

Therefore

$$
\begin{aligned}
& \left|\left[e_{c}\right]_{j}(k+1)\right| \\
& =\mid\left(1-s_{j}(k) \frac{\left[\hat{f}_{j}^{1}(k)\right]^{2}}{\left[\hat{f}_{j}^{1}(k)\right]^{2}+\alpha}\right) \\
& \times\left(\Delta r_{j}(k+1)+\left[e_{c}\right]_{j}(k)\right)+e_{j}(k+1) \\
& \leq\left|\left[e_{c}\right]_{j}(k)\left(1-s_{j}(k) \frac{\left[\widehat{f}_{j}^{1}(k)\right]^{2}}{\left[\widehat{f}_{j}^{1}(k)\right]^{2}+\alpha}\right)\right| \\
& +\mid \Delta r_{j}(k+1)\left(1-s_{j}(k) \frac{\left[\hat{f}_{j}^{1}(k)\right]^{2}}{\left[\hat{f}_{j}^{1}(k)\right]^{2}+\alpha}\right) \\
& +e_{j}(k+1) \\
& \leq\left|\left[e_{c}\right]_{j}(k)\right| \cdot\left|1-s_{j}(k) \frac{\left[\widehat{f}_{j}^{1}(k)\right]^{2}}{\left[\widehat{f}_{j}^{1}(k)\right]^{2}+\alpha}\right| \\
& +|\Delta r| \cdot\left|1-s_{j}(k) \frac{\left[\widehat{f}_{j}^{1}(k)\right]^{2}}{\left[\widehat{f}_{j}^{1}(k)\right]^{2}+\alpha}\right| \\
& +\left|e_{j}(k+1)\right| \text {. }
\end{aligned}
$$

From (A.24), one has

$$
\begin{aligned}
\left|\left[e_{c}\right]_{j}(k+1)\right| \leq & \left|\left[e_{c}\right]_{j}(k)\right| \cdot\left|1-s_{j}(k) \frac{\left[\hat{f}_{j}^{1}(k)\right]^{2}}{\left[\hat{f}_{j}^{1}(k)\right]^{2}+\alpha}\right| \\
& +r_{0} \cdot\left|1-s_{j}(k) \frac{\left[\widehat{f}_{j}^{1}(k)\right]^{2}}{\left[\widehat{f}_{j}^{1}(k)\right]^{2}+\alpha}\right| \\
& +\frac{2\left[e_{j}^{o}\right]_{\max }}{2-\left[\rho_{j}\right]_{\max }} \\
= & k_{1}\left|\left[e_{c}\right]_{j}(k)\right|+k_{2} .
\end{aligned}
$$

Since $0 \leq k_{1}<1$ and $k_{2}$ is bounded, according to [14, Lemma 13.1], one concludes that, using the control law (15), the solutions of error system (17) are UUB for all $k$ with ultimate bound $\lim _{k \rightarrow \infty}\left|\left[e_{c}\right]_{j}(k)\right| \leq\left(k_{2} /\left(1-k_{1}\right)\right)$.

\section{References}

[1] S. A. Billings and H. Nicholson, "Temperature weighting adaptive controller for electric arc furnaces," Ironmaking and Steelmaking, vol. 4, no. 4, pp. 216-221, 1977.

[2] M. Zhizhong and L. Jian, "Adaptive controller of an electric arc furnace with feedforward," Journal of Northeastern University, vol. 17, no. 1, pp. 65-68, 1996.

[3] A. S. Hauksdóttir, A. Gestsson, and A. Vésteinsson, "Current control of a three-phase submerged arc ferrosilicon furnace," Control Engineering Practice, vol. 10, no. 4, pp. 457-463, 2002.

[4] W. E. Staib and R. R. Staib, “The Intelligence arc furnace controller: a neural network electrode position optimization system for the electric arc furnace," in Proceedings of the IEEE International Joint Conference on Neural Network, vol. 3, pp. 19, Baltimore, Md, USA.

[5] W. E. Staib and N. G. Bliss, "Neural network control system for electric arc furnaces," Metallurgical Plant and Technology International, vol. 18, no. 2, p. 3, 1995.

[6] G. Ping, J.-C. Li, and X.-H. Liu, "Direct adaptive fuzzy sliding mode control of Arc furnace electrode regulator system," in Proceedings of the Chinese Control and Decision Conference (CCDC '09), pp. 2776-2781, Guilin, China, June 2009.

[7] S.-D. Zhang, "Decoupling control for electrode system in electric arc furnace based on neural network inverse identification," in Proceedings of the 6th International Conference on Intelligent Systems Design and Applications (ISDA '06), pp. 112-116, Jinan, China, October 2006.

[8] S. Zhang, K. Li, P. Li, and Z. Zhang, "Application of the electrode system intelligent controller for ladle furnace," in Proceedings of the IEEE International Conference on Mechatronics and Automation (ICMA '07), pp. 2570-2574, Harbin, China, August 2007.

[9] S. Zhang and X. Zheng, "Application of double model control scheme based on RBF inverse identification in electrode system of electrical arc furnace," in Proceedings of the IEEE International Conference on Automation and Logistics (ICAL '07), pp. 485489, Jinan, China, August 2007. 
[10] L. Li and Z. Mao, "A direct adaptive controller for EAF electrode regulator system using neural networks," Neurocomputing, vol. 82, pp. 91-98, 2012.

[11] C. Xinming, "The dynamic analysis for hydraulic system of EAF,' Industrial Heating, vol. 3, pp. 1-5, 1980.

[12] A. M. Valderhaug, Modelling and control of submerged-arc ferrosilicon furnaces [Ph.D. thesis], Norwegian Institute of Technology, Trondheim, Norway, report 92-81-W, 1992.

[13] R. P. Paiva, "Modelling and control of an electirc arc furance," Department of Automatic Control Lund Institute of Technology, Lund, Sweden, 1996.

[14] S. S. Ge, J. Zhang, and T. H. Lee, "Adaptive MNN control for a class of non-affine NARMAX systems with disturbances," Systems \& Control Letters, vol. 53, no. 1, pp. 1-12, 2004.

[15] O. Adetona, S. Sathananthan, and L. H. Keel, "Robust adaptive control of nonaffine nonlinear plants with small input signal changes," IEEE Transactions on Neural Networks, vol. 15, no. 2, pp. 408-416, 2004.

[16] O. Adetona, E. Garcia, and L. H. Keel, "A new method for the control of discrete nonlinear dynamic systems using neural networks," IEEE Transactions on Neural Networks, vol. 11, no. 1, pp. 102-112, 2000.

[17] D. Schroder, Intelligence Observer and Control Design for Nonlinear Systems, Springer, Berlin, Germany, 2000.

[18] M. R. Cowper, B. Mulgrew, and C. P. Unsworth, "Nonlinear prediction of chaotic signals using a normalised radial basis function network," Signal Processing, vol. 82, no. 5, pp. 775-789, 2002.

[19] G. Bugmann, "Normalized Gaussian radial basis function networks," Neurocomputing, vol. 20, no. 1-3, pp. 97-110, 1998.

[20] M. Benaim, "On the functional approximation with normalized Gaussian units," Neural Computation, vol. 6, no. 2, pp. 314-333, 1994.

[21] J. T. Spooner, M. Maggiore, and K. M. Passino, Stable Adaptive Control and Estimation for Nonlinear Systems, Wiley, New York, NY, USA, 2002. 


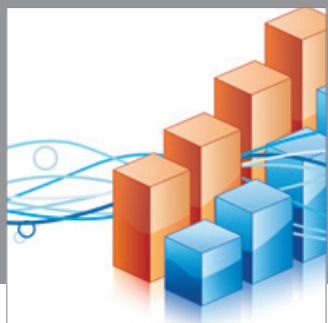

Advances in

Operations Research

mansans

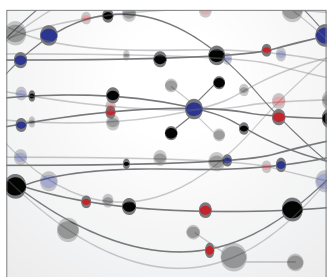

The Scientific World Journal
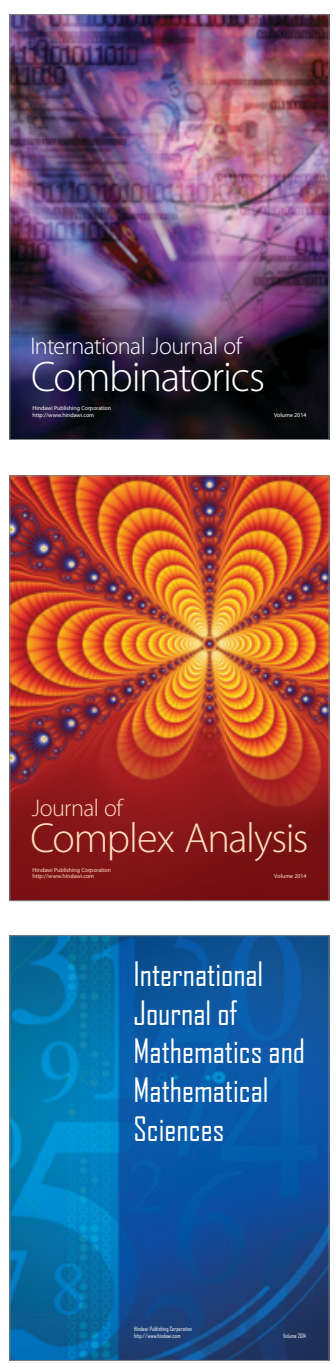


Submit your manuscripts at http://www.hindawi.com
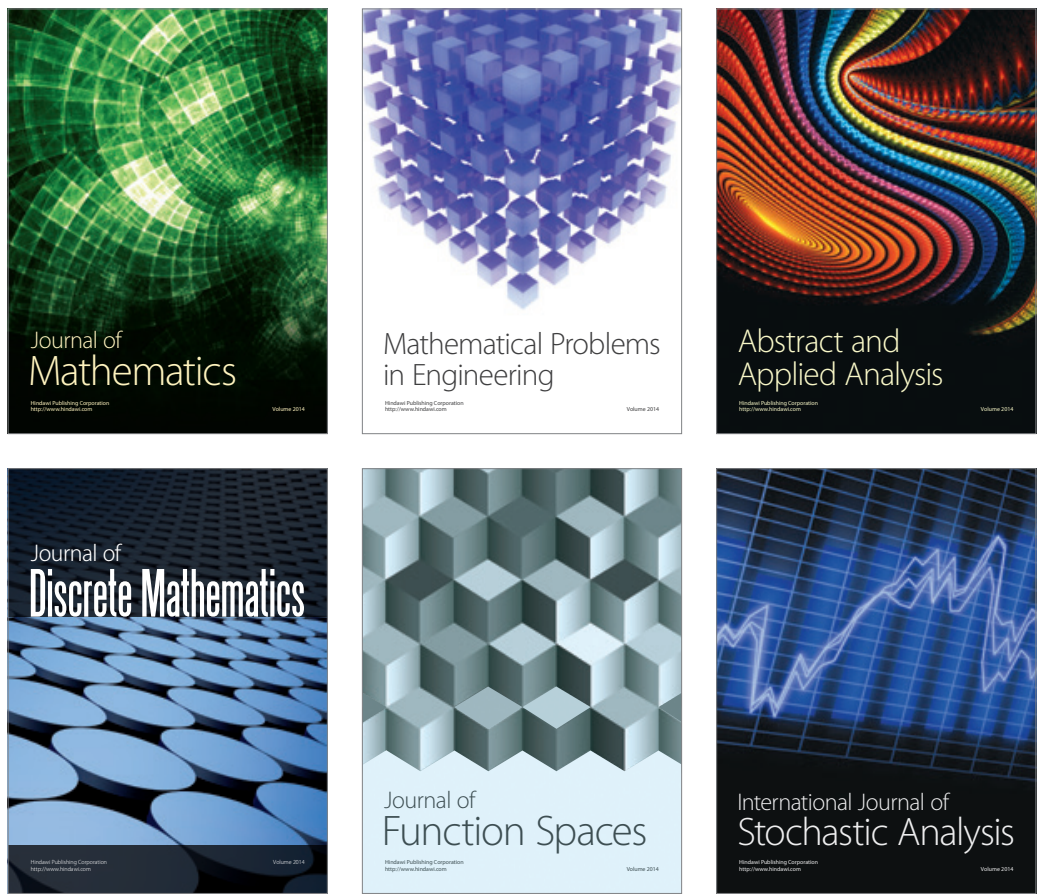

Journal of

Function Spaces

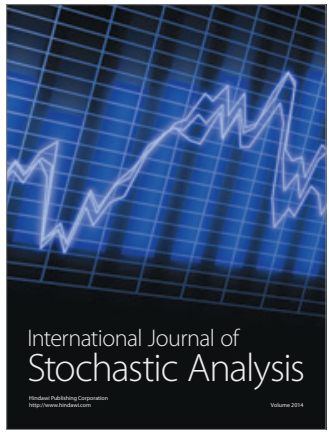

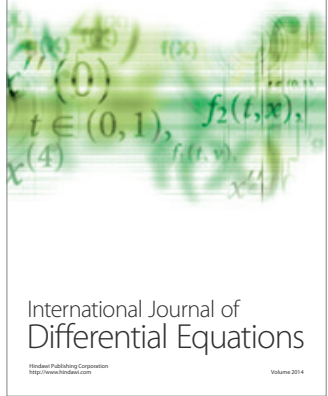
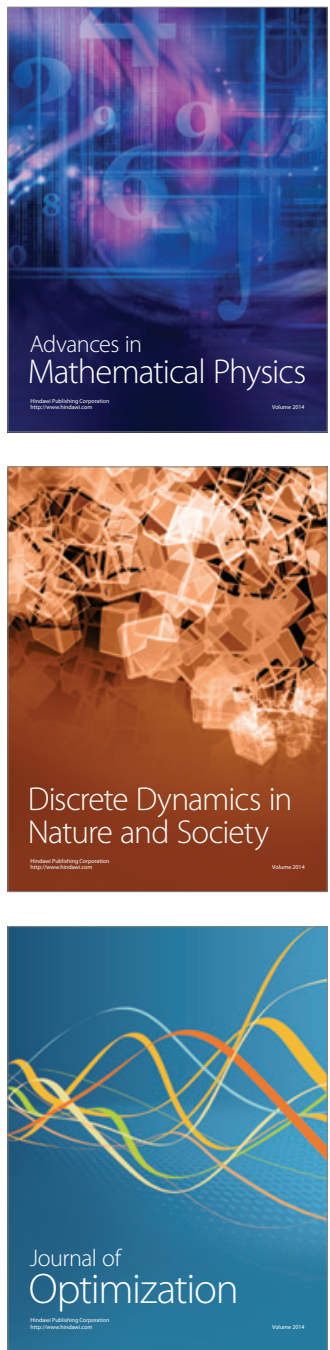